\title{
Inclusive Search for Standard Model Higgs Boson Production in the $W W$ Decay Channel Using the CDF II Detector
}

T. Aaltonen, ${ }^{24}$ J. Adelman, ${ }^{14}$ B. Álvarez González, ${ }^{12, \mathrm{x}}$ S. Amerio, ${ }^{44 \mathrm{~b}, 44 \mathrm{a}}$ D. Amidei, ${ }^{35}$ A. Anastassov, ${ }^{39}$ A. Annovi, ${ }^{20}$ J. Antos, ${ }^{15}$ G. Apollinari, ${ }^{18} \mathrm{~J}$. Appel,${ }^{18}$ A. Apresyan, ${ }^{49}$ T. Arisawa, ${ }^{58}$ A. Artikov, ${ }^{16} \mathrm{~J}$. Asaadi, ${ }^{54} \mathrm{~W}$. Ashmanskas,${ }^{18}$ A. Attal, ${ }^{4}$ A. Aurisano, ${ }^{54}$ F. Azfar, ${ }^{43}$ W. Badgett, ${ }^{18}$ A. Barbaro-Galtieri, ${ }^{29}$ V. E. Barnes, ${ }^{49}$ B. A. Barnett, ${ }^{26}$ P. Barria, ${ }^{47 c, 47 a}$ P. Bartos, ${ }^{15}$ G. Bauer, ${ }^{33}$ P.-H. Beauchemin, ${ }^{34}$ F. Bedeschi, ${ }^{47 a}$ D. Beecher, ${ }^{31}$ S. Behari ${ }^{26}$ G. Bellettini, ${ }^{47 b, 47 a}$ J. Bellinger, ${ }^{60}$ D. Benjamin, ${ }^{17}$ A. Beretvas, ${ }_{18}^{18}$ A. Bhatti, ${ }^{51}$ M. Binkley, ${ }^{18}$ D. Bisello, ${ }^{44 b, 44 a}$ I. Bizjak, ${ }^{31, e e}$ R. E. Blair, ${ }^{2}$ C. Blocker, ${ }^{7}$ B. Blumenfeld, ${ }^{26}$ A. Bocci,${ }^{17}$ A. Bodek,${ }^{50}$ V. Boisvert,${ }^{50}$ D. Bortoletto, ${ }^{49}$ J. Boudreau, ${ }^{48}$ A. Boveia, ${ }^{11}$ B. Brau, ${ }^{11, b}$ A. Bridgeman, ${ }^{25}$ L. Brigliadori, ${ }^{6 b, 6 a}$ C. Bromberg, ${ }^{36}$ E. Brubaker, ${ }^{14}$ J. Budagov, ${ }^{16}$ H. S. Budd, ${ }^{50}$ S. Budd ${ }^{25}$ K. Burkett, ${ }^{18}$ G. Busetto, ${ }^{44 b, 44 a}$ P. Bussey, ${ }^{22}$ A. Buzatu, ${ }^{34}$ K. L. Byrum, ${ }^{2}$ S. Cabrera, ${ }^{17, z}$ C. Calancha, ${ }^{32}$ S. Camarda, ${ }^{4}$ M. Campanelli, ${ }^{31}$ M. Campbell, ${ }^{35}$ F. Canelli, ${ }^{14,18}$ A. Canepa,${ }^{46}$ B. Carls,${ }^{25}$ D. Carlsmith,${ }^{60}$ R. Carosi ${ }^{47 a}$ S. Carrillo, ${ }^{19, o}$ S. Carron, ${ }^{18}$ B. Casal, ${ }^{12}$ M. Casarsa, ${ }^{18}$ A. Castro, ${ }^{6 b, 6 a}$ P. Catastini, ${ }^{47 c, 47 a}$ D. Cauz,${ }^{55 a}$ V. Cavaliere,${ }^{47 c, 47 a}$ M. Cavalli-Sforza ${ }^{4}$ A. Cerri, ${ }^{29}$ L. Cerrito, ${ }^{31, \mathrm{r}}$ S. H. Chang, ${ }^{28}$ Y. C. Chen, ${ }^{1}$ M. Chertok, ${ }^{8}$ G. Chiarelli, ${ }^{47 a}$ G. Chlachidze, ${ }^{18}$ F. Chlebana, ${ }^{18}$ K. Cho, ${ }^{28}$ D. Chokheli, ${ }^{16}$ J. P. Chou, ${ }^{23}$ K. Chung, ${ }^{18, p}$ W. H. Chung, ${ }^{60}$ Y. S. Chung, ${ }^{50}$ T. Chwalek ${ }^{27}$ C. I. Ciobanu, ${ }^{45}$ M. A. Ciocci, ${ }^{47 c, 47 a}$ A. Clark, ${ }^{21}$ D. Clark, ${ }^{7}$ G. Compostella, ${ }^{44 a}$ M. E. Convery, ${ }^{18}$ J. Conway, ${ }^{8}$ M. Corbo, ${ }^{45}$ M. Cordelli, ${ }^{20}$ C. A. Cox,${ }^{8}$ D. J. Cox,${ }^{8}$ F. Crescioli, ${ }^{47 b, 47 a}$ C. Cuenca Almenar, ${ }^{61}$ J. Cuevas, ${ }^{12, x}$ R. Culbertson, ${ }^{18}$ J. C. Cully, ${ }^{35}$

D. Dagenhart, ${ }^{18}$ N. d'Ascenzo, ${ }^{45, w}$ M. Datta, ${ }^{18}$ T. Davies,${ }^{22}$ P. de Barbaro, ${ }^{50}$ S. De Cecco, ${ }^{52 a}$ A. Deisher, ${ }^{29}$ G. De Lorenzo, M. Dell'Orso, ${ }^{47 b, 47 a}$ C. Deluca, ${ }^{4}$ L. Demortier,${ }^{51}$ J. Deng, ${ }^{17, g}$ M. Deninno, ${ }^{6 a}$ M. d'Errico, ${ }^{44 b, 44 a}$ A. Di Canto, ${ }^{47 b, 47 a}$ B. Di Ruzza, ${ }^{47 a}$ J. R. Dittmann, ${ }^{5}$ M. D’Onofrio, ${ }^{4}$ S. Donati, ${ }^{47 b, 47 a}$ P. Dong, ${ }^{18}$ T. Dorigo, ${ }^{44 a}$ S. Dube, ${ }^{53}$ K. Ebina,${ }^{58}$ A. Elagin,${ }^{54}$ R. Erbacher ${ }^{8}$ D. Errede ${ }^{25}$ S. Errede, ${ }^{25}$ N. Ershaidat, ${ }^{45, d d}$ R. Eusebi,${ }^{54}$ H. C. Fang ${ }^{29}$ S. Farrington, ${ }^{43}$ W. T. Fedorko, ${ }^{14}$ R. G. Feild, ${ }^{61}$ M. Feindt, ${ }^{27}$ J. P. Fernandez ${ }^{32}$ C. Ferrazza, ${ }^{47 d, 47 a}$ R. Field, ${ }^{19}$ G. Flanagan, ${ }^{49, t}$ R. Forrest, ${ }^{8}$ M. J. Frank, ${ }^{5}$ M. Franklin, ${ }^{23}$ J. C. Freeman, ${ }^{18}$ I. Furic, ${ }^{19}$ M. Gallinaro, ${ }^{51}$ J. Galyardt, ${ }^{13}$ F. Garberson, ${ }^{11}$ J. E. Garcia, ${ }^{21}$ A. F. Garfinkel, ${ }^{49}$ P. Garosi, ${ }^{47 c, 47 a}$ H. Gerberich, ${ }^{25}$ D. Gerdes, ${ }^{35}$ A. Gessler, ${ }^{27}$ S. Giagu, ${ }^{52 b, 52 a}$ V. Giakoumopoulou, ${ }^{3}$ P. Giannetti, ${ }^{47 a}$ K. Gibson, ${ }^{48}$ J. L. Gimmell, ${ }^{50}$ C. M. Ginsburg, ${ }^{18}$ N. Giokaris, ${ }^{3}$ M. Giordani, ${ }^{55 b, 55 a}$ P. Giromini, ${ }^{20}$ M. Giunta, ${ }^{47 a}$ G. Giurgiu, ${ }^{26}$ V. Glagolev, ${ }^{16}$ D. Glenzinski, ${ }^{18}$ M. Gold,${ }^{38}$ N. Goldschmidt, ${ }^{19}$ A. Golossanov, ${ }^{18}$ G. Gomez, ${ }^{12}$ G. Gomez-Ceballos, ${ }^{33}$ M. Goncharov, ${ }^{33}$ O. González, ${ }^{32}$ I. Gorelov, ${ }^{38}$ A. T. Goshaw, ${ }^{17}$ K. Goulianos, ${ }^{51}$ A. Gresele, ${ }^{44 b, 44 a}$ S. Grinstein, ${ }^{4}$ C. Grosso-Pilcher, ${ }^{14}$ R. C. Group,${ }^{18}$ U. Grundler, ${ }^{25}$ J. Guimaraes da Costa, ${ }^{23}$ Z. Gunay-Unalan, ${ }^{36}$ C. Haber, ${ }^{29}$ S. R. Hahn, ${ }^{18}$ E. Halkiadakis,${ }^{53}$ B.-Y. Han, ${ }^{50}$ J. Y. Han,${ }^{50}$ F. Happacher ${ }^{20}$ K. Hara,${ }^{56}$ D. Hare,${ }^{53}$ M. Hare,${ }^{57}$ R. F. Harr, ${ }^{59}$ M. Hartz ${ }^{48}$ K. Hatakeyama, ${ }^{5}$ C. Hays,${ }^{43}$ M. Heck,${ }^{27}$ J. Heinrich, ${ }^{46}$ M. Herndon, ${ }^{60}$ J. Heuser, ${ }^{27}$ S. Hewamanage, ${ }^{5}$ D. Hidas, ${ }^{53}$ C. S. Hill, ${ }^{11, \mathrm{~d}}$ D. Hirschbuehl,${ }^{27}$ A. Hocker,${ }^{18}$ S. Hou, ${ }^{1}$ M. Houlden, ${ }^{30}$ S.-C. Hsu, ${ }^{29}$ R. E. Hughes,${ }^{40}$ M. Hurwitz, ${ }^{14}$ U. Husemann, ${ }^{61}$ M. Hussein, ${ }^{36}$ J. Huston, ${ }^{36}$ J. Incandela, ${ }^{11}$ G. Introzzi, ${ }^{47 a}$ M. Iori, ${ }^{52 b, 52 a}$ A. Ivanov, ${ }^{8, q}$ E. James, ${ }^{18}$ D. Jang, ${ }^{13}$ B. Jayatilaka, ${ }^{17}$ E. J. Jeon, ${ }^{28}$ M. K. Jha, ${ }^{6 a}$ S. Jindariani, ${ }^{18}$ W. Johnson, ${ }^{8}$ M. Jones ${ }^{49}$ K. K. Joo, ${ }^{28}$ S. Y. Jun, ${ }^{13}$ J. E. Jung,${ }^{28}$ T. R. Junk,${ }^{18}$ T. Kamon,${ }^{54}$ D. Kar, ${ }^{19}$ P.E. Karchin,${ }^{59}$ Y. Kato, ${ }^{42, \mathrm{n}}$ R. Kephart, ${ }^{18}$ W. Ketchum, ${ }^{14}$ J. Keung, ${ }^{46}$ V. Khotilovich, ${ }^{54}$ B. Kilminster, ${ }^{18}$ D. H. Kim, ${ }^{28}$ H. S. Kim, ${ }^{28}$ H. W. Kim,${ }^{28}$ J. E. Kim, ${ }^{28}$ M. J. Kim, ${ }^{20}$ S. B. Kim,${ }^{28}$ S. H. Kim ${ }^{56}$ Y. K. Kim,${ }^{14}$ N. Kimura,${ }^{58}$ L. Kirsch, ${ }^{7}$ S. Klimenko, ${ }^{19}$ K. Kondo, ${ }^{58}$ D. J. Kong, ${ }^{28}$ J. Konigsberg, ${ }^{19}$ A. Korytov, ${ }^{19}$ A. V. Kotwal, ${ }^{17}$ M. Kreps,${ }^{27}$ J. Kroll, ${ }^{46}$ D. Krop,${ }^{14}$ N. Krumnack,,${ }^{5}$ M. Kruse, ${ }^{17}$ V. Krutelyov, ${ }^{11}$ T. Kuhr, ${ }^{27}$ N. P. Kulkarni, ${ }^{59}$ M. Kurata, ${ }^{56}$ S. Kwang, ${ }^{14}$ A. T. Laasanen, ${ }^{49}$ S. Lami, ${ }^{47 a}$ S. Lammel, ${ }^{18}$ M. Lancaster, ${ }^{31}$ R. L. Lander, ${ }^{8}$ K. Lannon, ${ }^{40, v}$ A. Lath, ${ }^{53}$ G. Latino, ${ }^{47 c, 47 a}$ I. Lazzizzera, ${ }^{44 b, 44 a}$ T. LeCompte, ${ }^{2}$ E. Lee, ${ }^{54}$ H. S. Lee, ${ }^{14}$ J. S. Lee, ${ }^{28}$

S. W. Lee, ${ }^{54, y}$ S. Leone, ${ }^{47 a}$ J. D. Lewis, ${ }^{18}$ C.-J. Lin, ${ }^{29}$ J. Linacre, ${ }^{43}$ M. Lindgren, ${ }^{18}$ E. Lipeles, ${ }^{46}$ A. Lister,${ }^{21}$

D. O. Litvintsev, ${ }^{18}$ C. Liu, ${ }^{48}$ T. Liu, ${ }^{18}$ N. S. Lockyer, ${ }^{46}$ A. Loginov, ${ }^{61}$ L. Lovas, ${ }^{15}$ D. Lucchesi,,${ }^{44,44 a}$ J. Lueck,${ }^{27}$ P. Lujan, ${ }^{29}$

P. Lukens, ${ }^{18}$ G. Lungu, ${ }^{51}$ J. Lys,${ }^{29}$ R. Lysak, ${ }^{15}$ D. MacQueen,${ }^{34}$ R. Madrak,${ }^{18} \mathrm{~K}$. Maeshima, ${ }^{18} \mathrm{~K}$. Makhoul, ${ }^{33}$

P. Maksimovic ${ }^{26}$ S. Malde ${ }^{43}$ S. Malik,${ }^{31}$ G. Manca,${ }^{30, f}$ A. Manousakis-Katsikakis, ${ }^{3}$ F. Margaroli,${ }^{49}$ C. Marino, ${ }^{27}$ C. P. Marino, ${ }^{25}$ A. Martin, ${ }^{61}$ V. Martin, ${ }^{22,1}$ M. Martínez, ${ }^{4}$ R. Martínez-Ballarín, ${ }^{32}$ P. Mastrandrea,${ }^{52 a}$ M. Mathis,${ }^{26}$

M. E. Mattson, ${ }^{59}$ P. Mazzanti, ${ }^{6 a}$ K. S. McFarland,${ }^{50}$ P. McIntyre, ${ }^{54}$ R. McNulty, ${ }^{30, k}$ A. Mehta,${ }^{30}$ P. Mehtala, ${ }^{24}$

A. Menzione, ${ }^{47 a}$ C. Mesropian, ${ }^{51}$ T. Miao, ${ }^{18}$ D. Mietlicki, ${ }^{35}$ N. Miladinovic, ${ }^{7}$ R. Miller,${ }^{36}$ C. Mills, ${ }^{23}$ M. Milnik,${ }^{27}$ A. Mitra, ${ }^{1}$ G. Mitselmakher, ${ }^{19}$ H. Miyake, ${ }^{56}$ S. Moed ${ }^{23}$ N. Moggi, ${ }^{6 a}$ M. N. Mondragon, ${ }^{18, o}$ C. S. Moon,${ }^{28}$ R. Moore,${ }^{18}$ M. J. Morello, ${ }^{47 a}$ J. Morlock, ${ }^{27}$ P. Movilla Fernandez,${ }^{18}$ J. Mülmenstädt, ${ }^{29}$ A. Mukherjee, ${ }^{18}$ Th. Muller, ${ }^{27}$ P. Murat ${ }^{18}$ M. Mussini, ${ }^{6 b, 6 a}$ J. Nachtman, ${ }^{18, p}$ Y. Nagai, ${ }^{56}$ J. Naganoma, ${ }^{56}$ K. Nakamura, ${ }^{56}$ I. Nakano, ${ }^{41}$ A. Napier,${ }^{57}$ J. Nett, ${ }^{60}$ C. Neu, ${ }^{46, b b}$ M. S. Neubauer, ${ }^{25}$ S. Neubauer, ${ }^{27}$ J. Nielsen, ${ }^{29, h}$ L. Nodulman, ${ }^{2}$ M. Norman, ${ }^{10}$ O. Norniella, ${ }^{25}$ E. Nurse, ${ }^{31}$ 
L. Oakes, ${ }^{43}$ S. H. Oh, ${ }^{17}$ Y. D. Oh, ${ }^{28}$ I. Oksuzian, ${ }^{19}$ T. Okusawa, ${ }^{42}$ R. Orava,${ }^{24}$ K. Osterberg, ${ }^{24}$ S. Pagan Griso, ${ }^{44 b, 44 a}$ C. Pagliarone,${ }^{55 a}$ E. Palencia, ${ }^{18}$ V. Papadimitriou, ${ }^{18}$ A. Papaikonomou, ${ }^{27}$ A. A. Paramanov, ${ }^{2}$ B. Parks,${ }^{40}$ S. Pashapour, ${ }^{34}$ J. Patrick, ${ }^{18}$ G. Pauletta, ${ }^{55 b, 55 a}$ M. Paulini, ${ }^{13}$ C. Paus,${ }^{33}$ T. Peiffer, ${ }^{27}$ D. E. Pellett, ${ }^{8}$ A. Penzo, ${ }^{55 a}$ T. J. Phillips,${ }^{17}$ G. Piacentino, ${ }^{47 a}$ E. Pianori, ${ }^{46}$ L. Pinera, ${ }^{19}$ K. Pitts, ${ }^{25}$ C. Plager,${ }^{9}$ L. Pondrom,${ }^{60}$ K. Potamianos, ${ }^{49}$ O. Poukhov, ${ }^{16, a}$ F. Prokoshin, ${ }^{16, a a}$ A. Pronko, ${ }^{18}$ F. Ptohos, ${ }^{18, j}$ E. Pueschel, ${ }^{13}$ G. Punzi, ${ }^{47 b, 47 a}$ J. Pursley, ${ }^{60}$ J. Rademacker, ${ }^{43, d}$ A. Rahaman, ${ }^{48}$ V. Ramakrishnan, ${ }^{60}$ N. Ranjan, ${ }^{49}$ I. Redondo, ${ }^{32}$ P. Renton, ${ }^{43}$ M. Renz,${ }^{27}$ M. Rescigno, ${ }^{52 a}$ S. Richter, ${ }^{27}$ F. Rimondi, ${ }^{6 b, 6 a}$ L. Ristori, ${ }^{47 a}$ A. Robson, ${ }^{22}$ T. Rodrigo, ${ }^{12}$ T. Rodriguez,${ }^{46}$ E. Rogers, ${ }^{25}$ S. Rolli, ${ }^{57}$ R. Roser, ${ }^{18}$ M. Rossi, ${ }^{55 a}$ R. Rossin,${ }^{11}$ P. Roy, ${ }^{34}$ A. Ruiz, ${ }^{12}$ J. Russ, ${ }^{13}$ V. Rusu, ${ }^{18}$ B. Rutherford, ${ }^{18}$ H. Saarikko, ${ }^{24}$ A. Safonov, ${ }^{54}$ W. K. Sakumoto, ${ }^{50}$ L. Santi, ${ }^{55 b, 55 a}$ L. Sartori, ${ }^{47 a}$ K. Sato,${ }^{56}$ V. Saveliev, ${ }^{45, w}$ A. Savoy-Navarro, ${ }^{45}$ P. Schlabach,,${ }^{18}$ A. Schmidt, ${ }^{27}$ E. E. Schmidt, ${ }^{18}$ M. A. Schmidt, ${ }^{14}$ M. P. Schmidt,,${ }^{6, a}$ M. Schmitt,,${ }^{39}$ T. Schwarz,${ }^{8}$ L. Scodellaro, ${ }^{12}$ A. Scribano, ${ }^{47 c, 47 a}$ F. Scuri, ${ }^{47 a}$ A. Sedov, ${ }^{49}$ S. Seidel,${ }^{38}$ Y. Seiya, ${ }^{42}$ A. Semenov, ${ }^{16}$ L. Sexton-Kennedy, ${ }^{18}$ F. Sforza, ${ }^{47 b, 47 a}$ A. Sfyrla ${ }^{25}$ S. Z. Shalhout, ${ }^{59}$ T. Shears, ${ }^{30}$ P. F. Shepard, ${ }^{48}$ M. Shimojima, ${ }^{56, u}$ S. Shiraishi, ${ }^{14}$ M. Shochet,${ }^{14}$ Y. Shon, ${ }^{60}$ I. Shreyber, ${ }^{37}$ A. Simonenko, ${ }^{16}$ P. Sinervo, ${ }^{34}$ A. Sisakyan, ${ }^{16}$ A. J. Slaughter, ${ }^{18}$ J. Slaunwhite, ${ }^{40}$ K. Sliwa, ${ }^{57}$ J. R. Smith, ${ }^{8}$ F. D. Snider,${ }^{18}$ R. Snihur, ${ }^{34}$ A. Soha,${ }^{18}$ S. Somalwar, ${ }^{53}$ V. Sorin, ${ }^{4}$ P. Squillacioti,${ }^{47 c, 47 a}$ M. Stanitzki, ${ }^{61}$ R. St. Denis, ${ }^{22}$ B. Stelzer,${ }^{34}$ O. Stelzer-Chilton, ${ }^{34}$ D. Stentz, ${ }^{39}$ J. Strologas, ${ }^{38}$ G. L. Strycker, ${ }^{35}$ J. S. Suh ${ }^{28}$ A. Sukhanov, ${ }^{19}$ I. Suslov, ${ }^{16}$ A. Taffard, ${ }^{25, g}$ R. Takashima ${ }^{41}$ Y. Takeuchi, ${ }^{56}$ R. Tanaka, ${ }^{41}$ J. Tang, ${ }^{14}$ M. Tecchio, ${ }^{35}$ P. K. Teng, ${ }^{1}$ J. Thom, ${ }^{18, i}$ J. Thome, ${ }^{13}$ G. A. Thompson, ${ }^{25}$ E. Thomson, ${ }^{46}$ P. Tipton, ${ }^{61}$ P. Ttito-Guzmán, ${ }^{32}$ S. Tkaczyk, ${ }^{18}$ D. Toback,${ }^{54} \mathrm{~S}$. Tokar, ${ }^{15} \mathrm{~K}$. Tollefson, ${ }^{36} \mathrm{~T}$. Tomura, ${ }^{56}$ D. Tonelli, ${ }^{18}$ S. Torre,${ }^{20}$ D. Torretta, ${ }^{18}$ P. Totaro, ${ }^{55 b, 55 a}$ M. Trovato, ${ }^{47 d, 47 a}$ S.-Y. Tsai, ${ }^{1}$ Y. Tu, ${ }^{46}$ N. Turini,${ }^{47 c, 47 a}$ F. Ukegawa, ${ }^{56}$ S. Uozumi, ${ }^{28}$ N. van Remortel,,${ }^{24, \mathrm{c}}$ A. Varganov, ${ }^{35}$ E. Vataga, ${ }^{47 \mathrm{~d}, 47 \mathrm{a}}$ F. Vázquez, ${ }^{19,0}$ G. Velev, ${ }^{18} \mathrm{C}$. Vellidis, ${ }^{3}$ M. Vidal, ${ }^{32}$ I. Vila, ${ }^{12}$ R. Vilar, ${ }^{12}$ M. Vogel,${ }^{38}$ I. Volobouev, ${ }^{29, y}$ G. Volpi, ${ }^{47 b, 47 a}$ P. Wagner, ${ }^{46}$ R. G. Wagner, ${ }^{2}$ R. L. Wagner ${ }^{18}$

W. Wagner, ${ }^{27, c c}$ J. Wagner-Kuhr, ${ }^{27}$ T. Wakisaka, ${ }^{42}$ R. Wallny, ${ }^{9}$ S. M. Wang, ${ }^{1}$ A. Warburton, ${ }^{34}$ D. Waters,${ }^{31}$

M. Weinberger ${ }^{54}$ J. Weinelt, ${ }^{27}$ W. C. Wester III,${ }^{18}$ B. Whitehouse, ${ }^{57}$ D. Whiteson, ${ }^{46,9}$ A. B. Wicklund, ${ }^{2}$ E. Wicklund, ${ }^{18}$ S. Wilbur, ${ }^{14}$ G. Williams, ${ }^{34}$ H. H. Williams,${ }^{46}$ P. Wilson, ${ }^{18}$ B. L. Winer, ${ }^{40}$ P. Wittich,${ }^{18, i}$ S. Wolbers,${ }^{18}$ C. Wolfe,${ }^{14}$ H. Wolfe, ${ }^{40}$ T. Wright,${ }^{35} \mathrm{X}$. Wu, ${ }^{21}$ F. Würthwein, ${ }^{10}$ A. Yagil, ${ }^{10} \mathrm{~K}$. Yamamoto, ${ }^{42}$ J. Yamaoka, ${ }^{17}$ U. K. Yang, ${ }^{14, \mathrm{~s}}$ Y. C. Yang,${ }^{28}$ W. M. Yao, ${ }^{29}$ G. P. Yeh, ${ }^{18}$ K. Yi, ${ }^{18, p}$ J. Yoh, ${ }^{18}$ K. Yorita ${ }^{58}$ T. Yoshida, ${ }^{42, m}$ G. B. $Y u,{ }^{17}$ I. Yu, ${ }^{28}$ S. S. Yu, ${ }^{18}$ J. C. Yun, ${ }^{18}$ A. Zanetti, ${ }^{55 a}$ Y. Zeng, ${ }^{17}$ X. Zhang, ${ }^{25}$ Y. Zheng, ${ }^{9, e}$ and S. Zucchelli ${ }^{6 b, 6 a}$

\section{(CDF Collaboration)}

${ }^{1}$ Institute of Physics, Academia Sinica, Taipei, Taiwan 11529, Republic of China

${ }^{2}$ Argonne National Laboratory, Argonne, Illinois 60439, USA

${ }^{3}$ University of Athens, 15771 Athens, Greece

${ }^{4}$ Institut de Fisica d'Altes Energies, Universitat Autonoma de Barcelona, E-08193, Bellaterra (Barcelona), Spain

${ }^{5}$ Baylor University, Waco, Texas 76798, USA

${ }^{6 a}$ Istituto Nazionale di Fisica Nucleare Bologna, I-40127 Bologna, Italy

${ }^{6 \mathrm{~b}}$ University of Bologna, I-40127 Bologna, Italy

${ }^{7}$ Brandeis University, Waltham, Massachusetts 02254, USA

${ }^{8}$ University of California, Davis, Davis, California 95616, USA

${ }^{9}$ University of California, Los Angeles, Los Angeles, California 90024, USA

${ }^{10}$ University of California, San Diego, La Jolla, California 92093, USA

${ }^{11}$ University of California, Santa Barbara, Santa Barbara, California 93106, USA

${ }^{12}$ Instituto de Fisica de Cantabria, CSIC-University of Cantabria, 39005 Santander, Spain

${ }^{13}$ Carnegie Mellon University, Pittsburgh, Pennsylvania 15213, USA

${ }^{14}$ Enrico Fermi Institute, University of Chicago, Chicago, Illinois 60637, USA

${ }^{15}$ Comenius University, 84248 Bratislava, Slovakia; Institute of Experimental Physics, 04001 Kosice, Slovakia

${ }^{16}$ Joint Institute for Nuclear Research, RU-141980 Dubna, Russia

${ }^{17}$ Duke University, Durham, North Carolina 27708, USA

${ }^{18}$ Fermi National Accelerator Laboratory, Batavia, Illinois 60510, USA

${ }^{19}$ University of Florida, Gainesville, Florida 32611, USA

${ }^{20}$ Laboratori Nazionali di Frascati, Istituto Nazionale di Fisica Nucleare, I-00044 Frascati, Italy

${ }^{21}$ University of Geneva, CH-1211 Geneva 4, Switzerland

${ }^{22}$ Glasgow University, Glasgow G12 8QQ, United Kingdom

${ }^{23}$ Harvard University, Cambridge, Massachusetts 02138, USA

${ }^{24}$ Division of High Energy Physics, Department of Physics, University of Helsinki and Helsinki Institute of Physics, FIN-00014, Helsinki, Finland 
${ }^{25}$ University of Illinois, Urbana, Illinois 61801, USA

${ }^{26}$ The Johns Hopkins University, Baltimore, Maryland 21218, USA

${ }^{27}$ Institut für Experimentelle Kernphysik, Karlsruhe Institute of Technology, D-76131 Karlsruhe, Germany

${ }^{28}$ Center for High Energy Physics: Kyungpook National University, Daegu 702-701, Korea;

Seoul National University, Seoul 151-742, Korea;

Sungkyunkwan University, Suwon 440-746, Korea;

Korea Institute of Science and Technology Information, Daejeon 305-806, Korea;

Chonnam National University, Gwangju 500-757, Korea;

Chonbuk National University, Jeonju 561-756, Korea

${ }^{29}$ Ernest Orlando Lawrence Berkeley National Laboratory, Berkeley, California 94720, USA

${ }^{30}$ University of Liverpool, Liverpool L69 7ZE, United Kingdom

${ }^{31}$ University College London, London WC1E 6BT, United Kingdom

${ }^{32}$ Centro de Investigaciones Energeticas Medioambientales y Tecnologicas, E-28040 Madrid, Spain

${ }^{33}$ Massachusetts Institute of Technology, Cambridge, Massachusetts 02139, USA

${ }^{34}$ Institute of Particle Physics: McGill University, Montréal, Québec, Canada H3A 2T8;

Simon Fraser University, Burnaby, British Columbia, Canada V5A 1S6;

University of Toronto, Toronto, Ontario, Canada M5S 1A7;

and TRIUMF, Vancouver, British Columbia, Canada V6T 2A3

${ }^{35}$ University of Michigan, Ann Arbor, Michigan 48109, USA

${ }^{36}$ Michigan State University, East Lansing, Michigan 48824, USA

${ }^{37}$ Institution for Theoretical and Experimental Physics, ITEP, Moscow 117259, Russia

${ }^{38}$ University of New Mexico, Albuquerque, New Mexico 87131, USA

${ }^{39}$ Northwestern University, Evanston, Illinois 60208, USA

${ }^{40}$ The Ohio State University, Columbus, Ohio 43210, USA

${ }^{41}$ Okayama University, Okayama 700-8530, Japan

${ }^{42}$ Osaka City University, Osaka 588, Japan

${ }^{43}$ University of Oxford, Oxford OX1 3RH, United Kingdom

${ }^{44 \mathrm{a}}$ Istituto Nazionale di Fisica Nucleare, Sezione di Padova-Trento, I-35131 Padova, Italy

${ }^{44 b}$ University of Padova, I-35131 Padova, Italy

${ }^{45}$ LPNHE, Universite Pierre et Marie Curie/IN2P3-CNRS, UMR7585, Paris, F-75252 France

${ }^{46}$ University of Pennsylvania, Philadelphia, Pennsylvania 19104, USA

${ }^{47 a}$ Istituto Nazionale di Fisica Nucleare Pisa, I-56127 Pisa, Italy

${ }^{47 \mathrm{~b}}$ University of Pisa, I-56127 Pisa, Italy

${ }^{47 \mathrm{c}}$ University of Siena, I-56127 Pisa, Italy

${ }^{47 \mathrm{~d}}$ Scuola Normale Superiore, I-56127 Pisa, Italy

${ }^{48}$ University of Pittsburgh, Pittsburgh, Pennsylvania 15260, USA

${ }^{49}$ Purdue University, West Lafayette, Indiana 47907, USA

${ }^{50}$ University of Rochester, Rochester, New York 14627, USA

${ }^{51}$ The Rockefeller University, New York, New York 10021, USA

${ }^{52 \mathrm{a}}$ Istituto Nazionale di Fisica Nucleare, Sezione di Roma 1, I-00185 Roma, Italy

${ }^{52 \mathrm{~b}}$ Sapienza Università di Roma, I-00185 Roma, Italy

${ }^{53}$ Rutgers University, Piscataway, New Jersey 08855, USA

${ }^{54}$ Texas A\&M University, College Station, Texas 77843, USA

${ }^{55}$ a Istituto Nazionale di Fisica Nucleare Trieste/Udine, I-34100 Trieste, Italy

${ }^{55 \mathrm{~b}}$ University of Trieste/Udine, I-33100 Udine, Italy

${ }^{56}$ University of Tsukuba, Tsukuba, Ibaraki 305, Japan

${ }^{57}$ Tufts University, Medford, Massachusetts 02155, USA

${ }^{58}$ Waseda University, Tokyo 169, Japan

${ }^{59}$ Wayne State University, Detroit, Michigan 48201, USA

${ }^{60}$ University of Wisconsin, Madison, Wisconsin 53706, USA

${ }^{61}$ Yale University, New Haven, Connecticut 06520, USA

(Received 25 January 2010; published 12 February 2010)

We present a search for standard model (SM) Higgs boson production using $p \bar{p}$ collision data at $\sqrt{s}=1.96 \mathrm{TeV}$, collected with the CDF II detector and corresponding to an integrated luminosity of $4.8 \mathrm{fb}^{-1}$. We search for Higgs bosons produced in all processes with a significant production rate and decaying to two $W$ bosons. We find no evidence for SM Higgs boson production and place upper limits at the $95 \%$ confidence level on the SM production cross section $\left(\sigma_{H}\right)$ for values of the Higgs boson mass $\left(m_{H}\right)$ in the range from 110 to $200 \mathrm{GeV}$. These limits are the most stringent for $m_{H}>130 \mathrm{GeV}$ and are 1.29 above the predicted value of $\sigma_{H}$ for $m_{H}=165 \mathrm{GeV}$. 
The standard model (SM) of particle physics unifies the electromagnetic and weak interactions into a single electroweak theory. However, experimental evidence and calculations in the framework of the SM show a difference of orders of magnitude in the cross section of electromagnetic and weak interactions at low energy. This fundamental difference is explained by the masses of the weak $W$ and $\mathrm{Z}$ intermediate bosons that mediate the weak interactions. These massive bosons are a result of electroweak symmetry breaking, which in the SM occurs through the Higgs mechanism. This theory is directly testable by the experimental observation of the Higgs boson, which is one of the primary objectives of modern particle physics. The production of Higgs bosons is expected to be observable at the Tevatron [1] where the Higgs boson has a large enough cross section as calculated at next-to-next-to-leading order (NNLO) [2-4] for exclusions or evidence to be seen with current data sets.

In this Letter we present a search for the production of SM Higgs bosons with subsequent decay to two oppositely charged $W^{(*)}$ bosons, where the asterisk indicates that $W$ bosons can be virtual. This search is most sensitive at high SM Higgs boson mass, $m_{H}>135 \mathrm{GeV}$ [5], where the Higgs boson decay to $W$ bosons is dominant [6]. Previous published searches for high-mass SM Higgs bosons set upper limits at the $95 \%$ confidence level (C.L.) on the production cross section for a SM Higgs boson $\left(\sigma_{H}\right)$ of at best 1.7 times greater than the predicted value [7]. The D0 collaboration is concurrently reporting an updated search of comparable sensitivity in this channel [8]. The results presented here improve the sensitivity of previous published searches by including new data, new search topologies, and by performing an inclusive search for all SM Higgs boson production processes with significant rate: gluon-gluon fusion through virtual-quark loops $(\mathrm{ggH})[9,10]$; production in association with a $W$ or $Z$ vector boson (VH) [11-13]; and vector boson fusion (VBF) $[11,14]$. The SM values of $\sigma_{H}$ for these processes at $m_{H}=160 \mathrm{GeV}$ are $\sigma_{g g H}=0.439 \mathrm{pb}, \sigma_{W H}=$ $0.051 \mathrm{pb}, \sigma_{\mathrm{ZH}}=0.033 \mathrm{pb}$, and $\sigma_{\mathrm{VBF}}=0.039 \mathrm{pb}$. This inclusive search expands the acceptance by $50 \%$ for $m_{H}=$ $160 \mathrm{GeV}$ compared to searching for only the $\mathrm{g} g \mathrm{H}$ production process as done previously by CDF [7].

The CDF II detector consists of a solenoidal spectrometer with a silicon tracker and an open cell drift-chamber (COT) surrounded by calorimeters and muon detectors [15]. The geometry is characterized using the azimuthal angle $\phi$ and the pseudorapidity $\eta \equiv-\ln [\tan (\theta / 2)]$, where $\theta$ is the polar angle relative to the proton beam axis. Transverse energy, $E_{T}$, is defined to be $E \sin \theta$, where $E$ is the energy of an electromagnetic (EM) and hadronic calorimeter energy cluster. Transverse momentum, $p_{T}$, is the track momentum component transverse to the beam line.
This analysis uses physics objects identified as jets, electrons, and muons as well as the estimated missing transverse energy. Electron and muon candidates (called electrons and muons for simplicity) are typically identified using the COT and EM calorimeter or muon chambers, respectively, and are described in detail below. Jet candidates (jets) are measured using the calorimeter towers with corrections to improve the estimated energy [16] and are required to have a measured $E_{T}$ greater than $15 \mathrm{GeV}$ and $|\eta|<2.5$. The missing transverse energy vector, $\vec{E}_{T}$, is defined as the opposite of the vector sum of the $E_{T}$ of all calorimeter towers, corrected to produce the correct average calorimeter response to jets and for the calorimeter response to muons.

The search is based on the requirement that events contain two charged leptons resulting from the decays of the final-state vector bosons. These leptons have opposite charge except in the case of the VH channel where they can have the same charge. We also make requirements on the $\mathscr{E}_{T}$ (explained below), which is indicative of the presence of neutrinos, in opposite-charge dilepton events. The Higgs boson signature can also involve jets of hadrons produced from the decay of one of the vector bosons in the $\mathrm{VH}$ process, forward quarks in the VBF process, or from the radiation of gluons.

One lepton must be identified by a trigger which performs real time selection of electrons or muons. One electron trigger requires an EM energy cluster in the central calorimeter $(|\eta|<1.1)$ with $E_{T}>18 \mathrm{GeV}$ pointed to by a COT track with $p_{T}>8 \mathrm{GeV}$. A second electron trigger requires an EM energy cluster with $E_{T}>20 \mathrm{GeV}$ in the forward calorimeter $(1.2<|\eta|<2.0)$ and uncorrected $\not E_{T}>15 \mathrm{GeV}$. Muon triggers are based on track segments in the muon chambers that are matched to a COT track with $p_{T}>18 \mathrm{GeV}$. Trigger efficiencies are measured using samples of observed leptonic $Z$ decays [17].

The selected events consist primarily of background SM processes from three categories. The first category contains processes that like the signal result in two charged leptons, $\mathscr{E}_{T}$, and possible jets, such as two $W$ bosons contributed by direct production $(W W)$, production of two $Z$ bosons $(Z Z)$ where one $Z$ boson decays to neutrinos, and top-quark pair production $(t \bar{t})$ where the $W$ bosons from top-quark decay subsequently decay leptonically. The second category consists of processes such as Drell-Yan (DY) production with possible jets where the observed $\mathbb{E}_{T}$ originates from the mismeasurement of lepton or jet energies. Also in this category are $Z Z$ and $W Z$ production where one or more of the final-state charged leptons are unobserved. The third category includes $W+$ jets $(W j)$ and $W \gamma$ production where a final-state jet or gamma is misidentified as a charged lepton. 
Higgs boson candidates are selected and contributions from the last two categories of background are reduced by applying the following initial selection. At least one charged lepton is required to match the lepton found in the trigger and have $E_{T}\left(p_{T}\right)>20 \mathrm{GeV}$ for electrons (muons). The second charged lepton is required to have $E_{T}\left(p_{T}\right)>10 \mathrm{GeV}$ except in events with same charge leptons, where both leptons are required to have $E_{T}\left(p_{T}\right)>$ $20 \mathrm{GeV}$. To reduce backgrounds from processes resulting in objects misidentified as charged leptons from vector boson decay (fake leptons), we employ a modified version of the lepton identification strategy developed for the $W Z$ observation analysis $[7,18]$. Candidate leptons are separated into seven categories: two for electrons; four for muons; and one for isolated tracks that project to detector regions with insufficient calorimeter coverage for energy measurements. The electron categories are distinguished by whether the electron is found using the central or the forward calorimeter. Electrons are further purified by a likelihood selection based on track quality, trackcalorimeter matching, calorimeter energy, calorimeter profile shape, and isolation information. Two of the muon categories use muons found in the central or forward muon chambers and the other two use tracks consistent with originating from minimum ionizing particles in either the central or forward calorimeters. Leptons are selected to be isolated by requiring that the sum of the $E_{T}$ for the calorimeter towers (or for central muons the sum of track momenta) in a cone of $\Delta R=\sqrt{(\Delta \eta)^{2}+(\Delta \phi)^{2}}<0.4$ around the lepton is less than $10 \%$ of the electron $E_{T}$ (muon $p_{T}$ ). Backgrounds involving mismeasured $\not E_{T}$ are reduced by requiring $\mathscr{E}_{T \text {,rel }}>25 \mathrm{GeV}$ for dielectrons, dimuons or events involving isolated tracks and $\mathscr{E}_{T \text {,rel }}>$ $15 \mathrm{GeV}$ for electron-muon events, where $\mathbb{E}_{T \text {,rel }} \equiv \mathbb{E}_{T}$ if $\Delta \phi_{\mathbb{E}_{T},(\ell, \text { jet })}>\frac{\pi}{2}, \mathscr{E}_{T} \sin \Delta \phi_{\mathbb{E}_{T},(\ell, \text { jet })}$ if $\Delta \phi_{\mathbb{E}_{T},(\ell \text {,jet })}<\frac{\pi}{2}$, and $\Delta \phi_{\mathbb{E}_{T},(\ell \text {,jet })}$ is the angle between the $\overrightarrow{\mathbb{E}}_{T}$ direction and the nearest lepton or jet. The $\mathscr{E}_{T \text {,rel }}$ selection is not applied for same charge lepton events since mismeasured $\mathscr{E}_{T}$ backgrounds are not large. We reduce DY production and heavy flavor backgrounds by requiring that the invariant mass of the lepton pair be greater than $16 \mathrm{GeV}$.

We further subdivide the observed events into six analysis channels based on jet multiplicity, lepton categories, and lepton charge combinations. The division is designed to optimize the sensitivity to the various Higgs boson production mechanisms [19]. Five of the channels have signatures with opposite-charge leptons. Events with zero jets and two central leptons are most sensitive to the leading order (LO) $g g H$ process and have $W W$ production as the dominant background. Events with zero jets, in which one lepton is identified as a forward electron or forward minimum ionizing track have an additional significant background from fake lepton sources. As in the zero jet case, we define two categories with one jet which are additionally sensitive to $\mathrm{VH}$ and VBF Higgs boson production. Events with two or more jets with any combination of opposite-charge leptons can originate from any Higgs boson production process and have $t \bar{t}$ as the dominant background. To reduce the $t \bar{t}$ background we reject events with $b$-quark jets [20], which are identified by finding displaced vertices from tracks in the jets. We define a separate channel for same charge dilepton events with one or more jets. Here we exclude forward electrons as the charge misidentification rate in the forward region is high. This category consists of signal events from $\mathrm{VH}$ production, where one lepton originates from the associated vector boson decay, and $W j$ events, where the jet is misidentified as a lepton. In addition to extended acceptance for VH and VBF production the final two channels add search topologies which are new compared to Ref. [7].

The acceptances, efficiencies and kinematic properties of the signal and background processes are determined primarily using simulation. Events are simulated with the MC@NLO program for $W W$ [21], PYTHIA for $H \rightarrow W W^{(*)}$, DY, $W Z, Z Z$, and $t \bar{t}$ [22], and the generator described in Ref. [23] for $W \gamma$. The response of the CDF II detector is then estimated with a GEANT-based simulation [24]. The cross sections for each process are normalized to NNLO calculations with logarithmic resummation $(\mathrm{ggH}[9,10])$, NNLO (VH [11-13] and $t \bar{t}$ for a top-quark mass of $172.4 \mathrm{GeV}$ [25]), and next-to-leading order calculations (VBF [11,14], $W W$ [21], $W Z$ and $Z Z$ [26], and $W \gamma$ [27]). Efficiency corrections for the simulated CDF II detector response for lepton, photon conversion, and $b$-jet reconstruction and identification are determined using samples of observed $Z \rightarrow \ell^{+} \ell^{-}$, photon conversions, and $b$-jets events, respectively. The probability that a jet will be misidentified as a lepton is measured using a sample of observed events collected with jet-based triggers and corrected for the contributions of leptons from $W$ and $Z$ decays. These probabilities are applied to each jet in a $W j$ enriched sample to estimate the number of $W j$ events that pass the selection [28].

Based on the selection described above we expect $594 \pm$ $63 W W, 97 \pm 13 W Z$ and $Z Z, 196 \pm 32 t \bar{t}, 339 \pm 61 \mathrm{DY}$, and $404 \pm 72 W \gamma$ and $W j$ events, for a total of $1630 \pm 140$ estimated background events. As an example, for a SM Higgs boson with $m_{H}=160 \mathrm{GeV}$ we expect $21.5 \pm$ $4.7 \mathrm{ggH}, 4.38 \pm 0.57 \mathrm{WH}, 1.59 \pm 0.21 \mathrm{ZH}$, and $1.61 \pm$ $0.26 \mathrm{VBF}$ events, for a total of $29.1 \pm 4.9$ Higgs boson events. We observe 1648 events. The indicated uncertainties are systematic and are described below.

After the initial selection the proportion of expected signal versus background is not sufficient to allow a significant result to be extracted quantifying the amount of signal present. Discrimination of signal from background is greatly enhanced by employing multivariate techniques. We train neural networks (NNs) using the NEUROBAYES [29] program with a combination of background events and simulated signal events for each analysis channel and at 


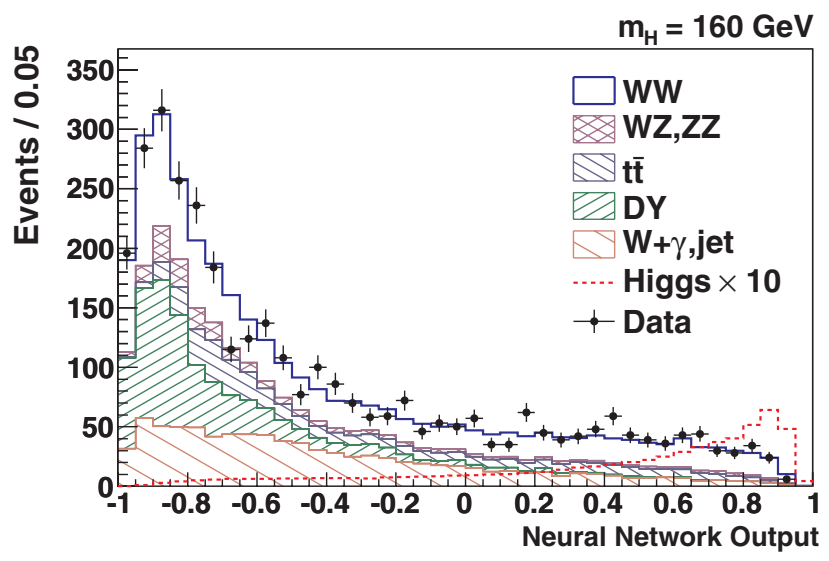

FIG. 1 (color online). The combined distribution of NN scores for backgrounds and a $m_{H}=160 \mathrm{GeV}$ Higgs boson compared to the observed data shown with statistical uncertainties. The Higgs boson distribution is normalized to 10 times the SM expectation.

each of 14 hypothesized $m_{H}$ values in the range $110 \leq$ $m_{H} \leq 200 \mathrm{GeV}$. The inputs to the NNs are based on kinematic quantities selected to exploit features such as the spin correlation between the $W$ bosons in Higgs boson decay, which results in the charged leptons from the $W$ decays tending to be more collinear than in $W W$ events, the presence of large $\mathbb{E}_{T}$ from the neutrinos, the transverse mass of the Higgs boson, which can be reconstructed from the leptons' four-momenta and $\vec{E}_{T}$, and the modest total energy of the Higgs boson decay products compared to $t \bar{t}$ decay [19]. In the zero jet categories we additionally classify each event by evaluating the observed kinematic configuration in a likelihood ratio of the signal probability density divided by the sum of the signal and background probability densities. These probability densities are determined from LO matrix element calculations of the cross sections $[18,28]$.

An example NN discriminant distribution for the combination of all categories is shown in Fig. 1. Signal and the a priori background expectations for a $160 \mathrm{GeV}$ Higgs boson are shown compared to the observed data.

We do not observe a significant excess of events and set upper limits at the $95 \%$ C.L. on $\sigma_{H}$, expressed as a ratio to the expected SM rate as a function of $m_{H}$. We employ a Bayesian technique [30] using a likelihood function constructed from the joint Poisson probability of observing the data in each bin of the discriminant $\mathrm{NN}$ output variables in each channel, integrating over the uncertainties of the normalization parameters using Gaussian priors. A constant prior in the signal rate is assumed.

When setting these limits we consider a variety of possible systematic effects including both those that change the normalization and those that change the shape of the kinematic distributions. The dominant systematic uncertainties are those on the theory predictions for the cross sections of signal and background processes and for the data driven background estimate used for $W j$. In addition, we consider the effect of variations from choices of renormalization and factorization scales, parton distribution function uncertainties, and differences between LO and higher order calculations on the acceptance of signal and background processes. The uncertainties on $\sigma_{H}$ are $5 \%$ for $W H$ and $Z H$, and $10 \%$ for VBF. We estimate an additional channel-dependent uncertainty for the $g g H$ process of approximately $7 \%-70 \%$, to account for scale variation cross section and acceptance uncertainties as a function of the number of identified jets, and a gluon PDF error of $8 \%$ following phenomenological NNLO studies [31]. The cross section uncertainties are $6 \%$ for diboson production, $10 \%$ for $t \bar{t}$ production, and $5 \%$ for DY production. We estimate an acceptance uncertainty to account for kinematic differences between generating at LO and higher order of $10 \%$ for all simulated processes except $W W$, DY, and $g g H$. We simulate the $W W$ process at higher order and assess a smaller uncertainty of 5\%. The jet multiplicity and $\mathscr{E}_{T}$ distributions for the DY process are not well modeled by the simulation and we assess uncertainties from $17 \%-32 \%$ depending on channel. We assess uncertainties of $20 \%$ and approximately $20 \%-30 \%$, depending on channel, on $W \gamma$ and $W j$ backgrounds, respectively, due to our modeling of conversion and fake lepton backgrounds. We also consider uncertainties on lepton identification and trigger efficiencies, which range from $1.4 \%$ to $3.4 \%$. Finally, we assess a $5.9 \%$ uncertainty on the integrated luminosity.

In Table I and Fig. 2 we show the median expected and observed upper limits on $\sigma_{H}$ for $14 m_{H}$ hypotheses calculated using the techniques and uncertainties explained above for the combination of all analysis categories.

In conclusion, we have performed an inclusive search for SM Higgs boson production in the two $W$ boson decay

TABLE I. Median expected and observed 95\% C.L. upper limits on $\sigma_{H}$ presented as a ratio to the predicted SM values of $\sigma_{H}$ as a function of $m_{H}$.

\begin{tabular}{|c|c|c|c|c|c|c|c|}
\hline$m_{H}(\mathrm{GeV})$ & 110 & 120 & 130 & 140 & 145 & 150 & 155 \\
\hline Expected $/ \sigma_{\mathrm{SM}}$ & 26.27 & 8.85 & 4.41 & 2.85 & 2.43 & 2.05 & 1.67 \\
\hline Observed $/ \sigma_{\mathrm{SM}}$ & 38.89 & 12.04 & 6.38 & 4.21 & 3.23 & 2.62 & 2.04 \\
\hline$m_{H}(\mathrm{GeV})$ & 160 & 165 & 170 & 175 & 180 & 190 & 200 \\
\hline Expected $/ \sigma_{\mathrm{SM}}$ & 1.26 & 1.20 & 1.44 & 1.72 & 2.09 & 3.24 & 4.53 \\
\hline Observed $/ \sigma_{\mathrm{SM}}$ & 1.34 & 1.29 & 1.69 & 1.94 & 2.24 & 4.06 & 6.74 \\
\hline
\end{tabular}




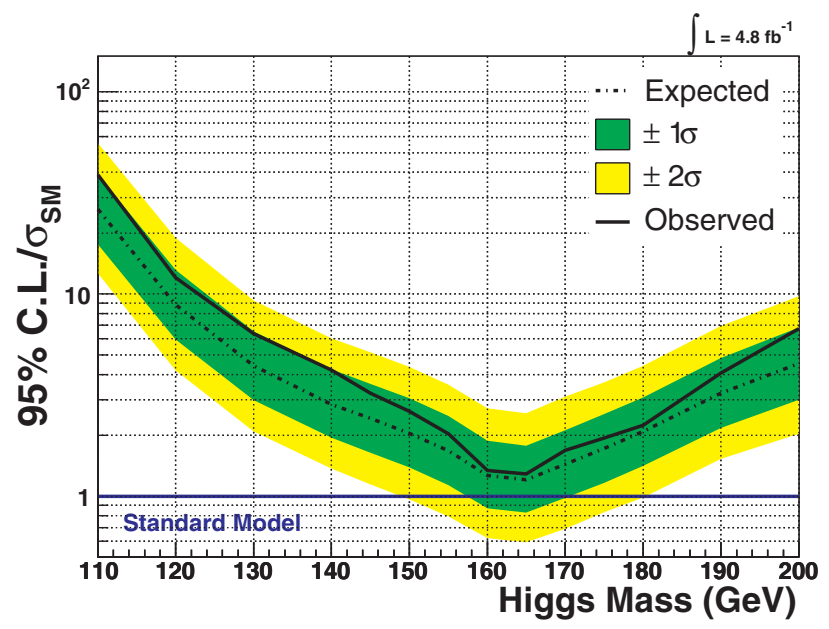

FIG. 2 (color online). Expected and observed upper limits at the $95 \%$ C.L. on $\sigma_{H}$ presented as a ratio to the predicted SM values as a function of $m_{H}$. The dashed line represents the median expected limits, the green and yellow bands the estimated one and two sigma probability bands for the distribution of expectations, and the solid line the observed limit.

mode where the final state contains two charged leptons. We observe no evidence for SM Higgs boson production and set upper limits on $\sigma_{H}$. These limits are the most stringent to date from a single experiment for high-mass SM Higgs boson production. We limit (at the 95\% C.L.) SM Higgs boson production to be no larger than 1.34 and 1.29 times the expected SM cross sections for $m_{H}=160$ and $m_{H}=165 \mathrm{GeV}$, respectively.

We thank the Fermilab staff and the technical staffs of the participating institutions for their vital contributions. This work was supported by the U.S. Department of Energy and National Science Foundation; the Italian Istituto Nazionale di Fisica Nucleare; the Ministry of Education, Culture, Sports, Science and Technology of Japan; the Natural Sciences and Engineering Research Council of Canada; the National Science Council of the Republic of China; the Swiss National Science Foundation; the A.P. Sloan Foundation; the Bundesministerium für Bildung und Forschung, Germany; the World Class University Program, the National Research Foundation of Korea; the Science and Technology Facilities Council and the Royal Society, UK; the Institut National de Physique Nucleaire et Physique des Particules/CNRS; the Russian Foundation for Basic Research; the Ministerio de Ciencia e Innovación, and Programa Consolider-Ingenio 2010, Spain; the Slovak R\&amp;D Agency; and the Academy of Finland.

\footnotetext{
${ }^{\mathrm{a}}$ Deceased

${ }^{\mathrm{b}}$ Visitor from University of Massachusetts Amherst, Amherst, MA 01003, USA.
}

${ }^{c}$ Visitor from Universiteit Antwerpen, B-2610 Antwerp, Belgium.

${ }^{\mathrm{d}}$ Visitor from University of Bristol, Bristol BS8 1TL, United Kingdom.

${ }^{e}$ Visitor from Chinese Academy of Sciences, Beijing 100864, China

${ }^{\mathrm{f}}$ Visitor from Istituto Nazionale di Fisica Nucleare, Sezione di Cagliari, 09042Monserrato (Cagliari), Italy.

${ }^{\mathrm{g}}$ Visitor from University of California Irvine, Irvine, CA 92697, USA.

${ }^{\mathrm{h}}$ Visitor from University of California Santa Cruz, Santa Cruz, CA 95064, USA.

${ }^{\mathrm{i}}$ Visitor from Cornell University, Ithaca, NY 14853, USA.

${ }^{\mathrm{j}}$ Visitor from University of Cyprus, Nicosia CY-1678, Cyprus.

${ }^{\mathrm{k}}$ Visitor from University College Dublin, Dublin 4, Ireland.

${ }^{1}$ Visitor from University of Edinburgh, Edinburgh EH9 3JZ, United Kingdom.

${ }^{\mathrm{m}}$ Visitor from University of Fukui, Fukui City, Fukui Prefecture, Japan 910-0017.

${ }^{\mathrm{n}}$ Visitor from Kinki University, Higashi-Osaka City, Japan 577-8502.

${ }^{\circ}$ Visitor from Universidad Iberoamericana, Mexico D.F., Mexico.

${ }^{\mathrm{p}}$ Visitor from University of Iowa, Iowa City, IA 52242, USA.

${ }^{\mathrm{q}}$ Visitor from Kansas State University, Manhattan, KS 66506, USA.

${ }^{\mathrm{r}}$ Visitor from Queen Mary, University of London, London, E1 4NS, United Kingdom.

${ }^{\mathrm{s}}$ Visitor from University of Manchester, Manchester M13 9PL, United Kingdom.

${ }^{t}$ Visitor from Muons, Inc., Batavia, IL 60510, USA.

${ }^{\text {u} V i s i t o r ~ f r o m ~ N a g a s a k i ~ I n s t i t u t e ~ o f ~ A p p l i e d ~ S c i e n c e, ~}$ Nagasaki, Japan.

${ }^{\mathrm{v}}$ Visitor from University of Notre Dame, Notre Dame, IN 46556, USA.

${ }^{\mathrm{w}}$ Visitor from Obninsk State University, Obninsk, Russia.

${ }^{\mathrm{x}}$ Visitor from University de Oviedo, E-33007 Oviedo, Spain.

${ }^{\mathrm{y}}$ Visitor from Texas Tech University, Lubbock, TX 79609 , USA.

${ }^{\mathrm{z}}$ Visitor from IFIC(CSIC-Universitat de Valencia), 56071 Valencia, Spain.

${ }^{\text {aa } V i s i t o r ~ f r o m ~ U n i v e r s i d a d ~ T e c n i c a ~ F e d e r i c o ~ S a n t a ~ M a r i a, ~}$ 110v Valparaiso, Chile.

${ }^{\mathrm{bb}}$ Visitor from University of Virginia, Charlottesville, VA 22906, USA.

${ }^{\mathrm{cc}}$ Visitor from Bergische Universität Wuppertal, 42097 Wuppertal, Germany.

${ }^{\mathrm{dd}}$ Visitor from Yarmouk University, Irbid 211-63, Jordan.

${ }^{\mathrm{ee}}$ On leave from J. Stefan Institute, Ljubljana, Slovenia.

[1] T. Han and R.-J. Zhang, Phys. Rev. Lett. 82, 25 (1999).

[2] R. V. Harlander and W. B. Kilgore, Phys. Rev. Lett. 88, 201801 (2002).

[3] C. Anastasiou and K. Melnikov, Nucl. Phys. B 646, 220 (2002). 
[4] V. Ravindran, J. Smith, and W. L. van Neerven, Nucl. Phys. B 665, 325 (2003).

[5] We use natural units where $c$ and $\hbar$ are taken as unity and momentum and mass are referred to in units of energy.

[6] A. Djouadi, J. Kalinowski, and M. Spira, Comput. Phys. Commun. 108, 56 (1998).

[7] T. Aaltonen et al. (CDF Collaboration), Phys. Rev. Lett. 102, 021802 (2009).

[8] V. M. Abazov et al. (D0 Collaboration), this issue, Phys. Rev. Lett. 104, 061804 (2010).

[9] D. de Florian and M. Grazzini, Phys. Lett. B 674, 291 (2009).

[10] C. Anastasiou, R. Boughezal, and F. Petriello, J. High Energy Phys. 04 (2009) 003.

[11] K. A. Assamagan et al. (Higgs Working Group), arXiv: hep-ph/0406152.

[12] O. Brein, A. Djouadi, and R. Harlander, Phys. Lett. B 579, 149 (2004).

[13] M. L. Ciccolini, S. Dittmaier, and M. Kramer, Phys. Rev. D 68, 073003 (2003).

[14] E. L. Berger and J. M. Campbell, Phys. Rev. D 70, 073011 (2004).

[15] D. Acosta et al. (CDF Collaboration), Phys. Rev. D 71, 032001 (2005).

[16] A. Bhatti et al., Nucl. Instrum. Methods Phys. Res., Sect. A 566, 375 (2006).

[17] A. Abulencia et al. (CDF Collaboration), J. Phys. G 34, 2457 (2007).
[18] A. Abulencia et al. (CDF Collaboration), Phys. Rev. Lett. 98, 161801 (2007).

[19] D. Hidas, Ph.D. thesis, Duke University [Fermilab Report No. FERMILAB-THESIS-2008-83, 2008].

[20] A. Abulencia et al. (CDF Collaboration), Phys. Rev. Lett. 97, 082004 (2006).

[21] S. Frixione and B. R. Webber, J. High Energy Phys. 06 (2002) 029.

[22] T. Sjostrand, S. Mrenna, and P. Skands, J. High Energy Phys. 05 (2006) 026.

[23] U. Baur and E. L. Berger, Phys. Rev. D 47, 4889 (1993).

[24] R. Brun, R. Hagelberg, M. Hansroul, and J. Lassalle, version 3.15, Report No. CERN-DD-78-2-REV.

[25] S. Moch and P. Uwer, Nucl. Phys. B, Proc. Suppl. 183, 75 (2008).

[26] J. M. Campbell and R. K. Ellis, Phys. Rev. D 60, 113006 (1999).

[27] U. Baur, T. Han, and J. Ohnemus, Phys. Rev. D 57, 2823 (1998).

[28] S.-C. Hsu, Ph.D. thesis, UC, San Diego [Fermilab Report No. FERMILAB-THESIS-2008-61, 2008].

[29] M. Feindt and U. Kerzel, Nucl. Instrum. Methods Phys. Res., Sect. A 559, 190 (2006).

[30] C. Amsler et al. (Particle Data Group), Phys. Lett. B 667, 1 (2008).

[31] C. Anastasiou, G. Dissertori, M. Grazzini, F. Stockli, and B. R. Webber, J. High Energy Phys. 08 (2009) 099. 